\title{
Acknowledgment to Reviewers of Instruments in 2021
}

\author{
Instruments Editorial Office
}

Citation: Instruments Editorial

Office. Acknowledgment to

Reviewers of Instruments in 2021.

Instruments 2022, 6, 8 .

https://doi.org/10.3390/

instruments6010008

Published: 26 January 2022

Publisher's Note: MDPI stays neutral with regard to jurisdictional claims in published maps and institutional affiliations.

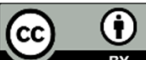

Copyright: $(2022$ by the authors. $\mathrm{Li}-$ censee MDPI, Basel, Switzerland. This article is an open access article distributed under the terms and conditions of the Creative Commons Attribution (CC BY) license (https://creativecommons.org/licenses/by/4.0/)
MDPI AG, St. Alban-Anlage 66, 4052 Basel, Switzerland

Rigorous peer-reviews are the basis of high-quality academic publishing. Thanks to the great efforts of our reviewers, Instruments was able to maintain its standards for the high quality of its published papers. Thanks to the contribution of our reviewers, in 2021, the median time to first decision was 20 days and the median time to publication was 44 days. The editors would like to extend their gratitude and recognition to the following reviewers for their precious time and dedication, regardless of whether the papers they reviewed were finally published:

Alejo, Aarón
Alessi, Antonino
Anfimov, Nikolay
Bay, Fatih
Baylac, Maud
Beznosko, Dmitriy
Bindra, Jasleen Kaur
Bonatto, Alexandre
Braccini, Saverio
Branca, Antonio
Brunbauer, Florian Maximilian
Burkhardt, Helmut
Caccia, Massimo
Canci, Nicola
Carasco, Cédric
Carosi, Gianpaolo P.
Casalbuoni, Sara
Cattaneo, Paolo W.
Cerulli, R.
Chen, Chiachung
Chen, June-Rong
Choi, Chang-Hoon
Colas, Paul
Constable, David A.
Cottle, Amy
Daví, Fabrizio
Dehmelt, Klaus
Demmel, Franz
Di Digioacchino, Daniele
Di Giovanni, Adriano
Di Mauro, Mattia
Duranti, Matteo
Elagin, Andrey L.
Faillace, Luigi

Finocchiaro, Paolo

Fiore, Salvatore

Friedman, Aron

Fursa, Dmitry

Gazis, Evangelos

Georges, Robert

Harnew, Neville

Heijne, Erik

Heikkinen, Pauli

Hinostroza Sáenz, Israel David

Irastorza, Igor García

Kasiński, Krzysztof

Khodja, Hicham

Kiang, Jean-Fu

Li, Derun

Loisch, Gregor

Marcatili, Sara

Maricic, Jelena

Matvienko, Dmitry

McCabe, Christopher

Menegolli, Alessandro

Mietelski, Jerzy Wojciech

Mozzanica, Aldo

Muggli, Patric

Nesteruk, Konrad P.

Penco, Giuseppe

Pérez-Hernández, Jose A.

Perez-Ramirez, Carlos

Pilot, Roberto

Posen, Sam

Rhee, Huinam

Roedel, Christian

Rossi, Andrea Renato

Sauli, Fabio 
Sedmik, René

Selvaraju, Ram Kumar

Skoczeń, Andrzej

Sokolova, Anna

Starodumov, Andrei

Tarantino, Gabriella

Tavakoli, Keihan
Tinti, Gemma

Tytgat, Michael

Vadrucci, Monia

Verwilligen, Piet

Westerdale, Shawn

Yeom, Jung Yeol 